\title{
A SYSTEM DYNAMICS MODEL OF TUBERCULOSIS DIFFUSION WITH RESPECT TO CONTACT TRACING INVESTIGATION
}

\author{
Yuan Tian \\ Fatima Alawami \\ Assaad Al-Azem \\ Nathaniel Osgood \\ Vernon Hoeppner \\ Christopher Dutchyn \\ University of Saskatchewan \\ 1437 College Drive \\ Saskatoon, SK, Canada
}

\begin{abstract}
Despite great efforts to control the spread of tuberculosis (TB), the disease remains stubbornly persistent, having infected one third of the world population, and causing more than 1.5 million deaths annually. To better understand the epidemiology of TB, past modeling efforts have sought to understand how TB prevention and control policies affect infection spread in the population. This paper describes a preliminary dynamic model to evaluate the role of current contact tracing policies in managing TB transmission. Through a novel representation of contact tracing dynamics, the model supports investigation of how TB outcomes are affected by changes to the breadth and timeliness of contact investigation. Model results suggest that while successful contact tracing is self-limiting, it plays a critical role in TB control. Results also suggest that expanded breadth of contact tracing offers diminishing returns, and underscores the desirability of highly targeted contact tracing and the desirability of richer models.
\end{abstract}

\section{INTRODUCTION}

Tuberculosis (TB) is a communicable disease which burdens public health systems across the globe. This burden has motivated diverse control and prevention programs to fight against the disease. Notable advances in immunology and microbiology have provided fundamental insights into the detailed mechanism of TB infection in the past century. At the same time, epidemiological modeling centered specifically on the dynamics of infections at the population level has profoundly enriched our understanding of the properties of TB and prevention (Anderson and May 1991, Hassmiller 2007). In spite of the remarkable insights gained from biological research and dynamic modeling, the TB incidence rate is still high in certain geographic and demographic zones. In the Canadian province of Saskatchewan, the Saskatchewan AntiTuberculosis League and the Provincial TB Control program made historical inroads against TB, but the TB incidence rate in the province remains among the highest across Canada (Osgood et al. 2011). It is still challenging to establish practical and efficient prevention policies.

In spite of the focus on understanding infectious disease dynamics within modeling applications, the need for integrating the dynamics of prevention strategies and control policies into epidemiological models has long been acknowledged. Both deterministic and stochastic models have been developed to evaluate the impact and efficiency of different control prevention and control programs for infectious disease, such as vaccination, screening, targeted treatment and contact tracing (Huerta and Tsimring 2002; Hy- 


\section{Tian, Alawami, Al-Azem, Osgood, Hoeppner, and Dutchyn}

man, Li, and Stanley 2003; Eames and Keeling 2003), but relatively few models integrate a representation of the dynamics of prevention and control program (White P.J. et al. 2005).

Although previous work has explored some theoretical aspects of different treatment and prevention strategies (Aparicio and Hernandez 2006; Ziv, Daley, and Blower 2001), they lack detailed representation of the ongoing operational processes of control programs. Specifically, it is necessary to characterize the dynamics of a combination of several important control strategies such as active diagnosis, treatment of latent TB infection (TLTBI) and contact tracing investigation.

In order to provide an illustration of how dynamic models can be used to evaluate and guide operational control strategies, we introduce a System Dynamics model of TB transmission that explores some approaches to integrating infection dynamics with a group of ongoing control policies, most notably contact tracing and TLTBI, across a community in the Canadian province of Saskatchewan.

To improve the quality and efficiency of disease control, sensitivity analysis can be conducted to sort through alternatives and to assist the decision making process in identifying the best choice with which to serve both financial constraints as well as objectives of intervention programs. We present and discuss two modeling questions to illustrate how System Dynamics models could be used to support and improve the development of control policies, contact tracing investigation strategy in particular. With respect to efficiency of contact tracing, our first question aims at exploring the efficiency in terms of investigating fraction of the contacts. This paper also seeks to explore the benefits conferred by speeding up the contact tracing investigation procedure. More detailed explanation regarding these two questions is demonstrated in Section 4. The experimental results are presented in Section 5. Section 6 discusses model findings and summarizes our work.

\section{TUBERCULOSIS PREVENTION AND CONTROL ACTIVITIES}

TB is the second largest cause of death from infectious disease across the world (Hassmiller 2007). Most TB bacteria are transmitted via airborne mechanisms, and while the bacteria can invade diverse organs, it most commonly infects lungs. A TB infection does not instantly bring on active TB disease; typically there is an incubation period before disease emerges. This period can be extremely prolonged: $90-95 \%$ of individuals live the remainder of their lives without developing active TB. During this incubation phase, the bacteria persists in an immunologically controlled state in what is known as latent TB.

The Tuberculosis Prevention and Control Unit of Health Canada has implemented prevention and control programs in an attempt to eliminate Tuberculosis (Al-Azem 2007), including vaccination, active TB treatment, treatment for latent TB infection, screening programs, and contact tracing investigation.

In particular, contact tracing investigation, an integral part of disease control programs, aims at finding latently infected people and active (and especially infectious) TB cases. Contact tracing investigation in Saskatchewan targets two classes of people, namely infectious TB cases and primary TB cases. Infectious TB cases are transmitters of the infection; investigating infectious TB cases can eventually help find more infected persons with high risk of developing TB in their lifetime. Providing treatment for latently infected people can lower their risk of developing active TB and disrupt the potential pathways of TB transmission. The term "reverse contact tracing" describes the investigation of primary TB cases who are infected within 2 years. This procedure can help identify the sources of the infection.

In addition, as part of TB investigation, a Mantoux test is used to check whether or not a client is infected. A positive result suggests that the client is infected by TB bacteria; otherwise, the risk of active TB disease is ruled out. In contact tracing and screening, the Mantoux test is used to check whether an individual is infected.

\section{SYSTEM DYNAMICS MODEL OF TUBERCULOSIS TRANSMISSION}

We have developed a System Dynamics model of TB transmission to reflect the contact tracing investigation in community 1 . Since most of people in community 1 live on reserve, immigration is restricted. It is therefore assumed that the population changes only via birth or death. 


\subsection{Structure of System Dynamics TB Model}

Our model extends existing mathematical models of TB dynamics (Anderson and May 1991, Osgood et al. 2011) to capture the dynamics of TB in the context of a community as well as to estimate the efficiency of the current contact tracing program. While the model in current contribution is parameterized with and calibrated to a particular locality, the model is rather stylized. For example, the model does not capture certain important risk factors known within the epidemiological context, such as age groups. The quantitative results of the model for TB levels are therefore to be taken with a significant grain of salt. We instead believe that the primary contributions of this study lie in the structural innovations used to capture contact tracing at an aggregated level.

The structure of our model is illustrated by stocks and flows in Figure 1. Our model consists of eleven stocks. Of these eleven stocks, ten represent the population, distinguishing individuals according to TBspecific health status and status with respect to contact tracing. The remaining stock named "Named By TB Cases for Investigation (Cv)" represents a name list of contacts to be investigated via contact tracing. It is worth emphasizing that this stock does not represent on segment of the population collectively represented the other stocks. There are no individuals in this stock - rather, the stock holds a list of names of the contacts which are obtained from those traced active TB cases. The named individuals are assumed to reside in one of the other 10 stocks. Transitions depicted by flows in the upper part of the diagram reflect the pathways through which people's health and contact tracing status can progress over time.

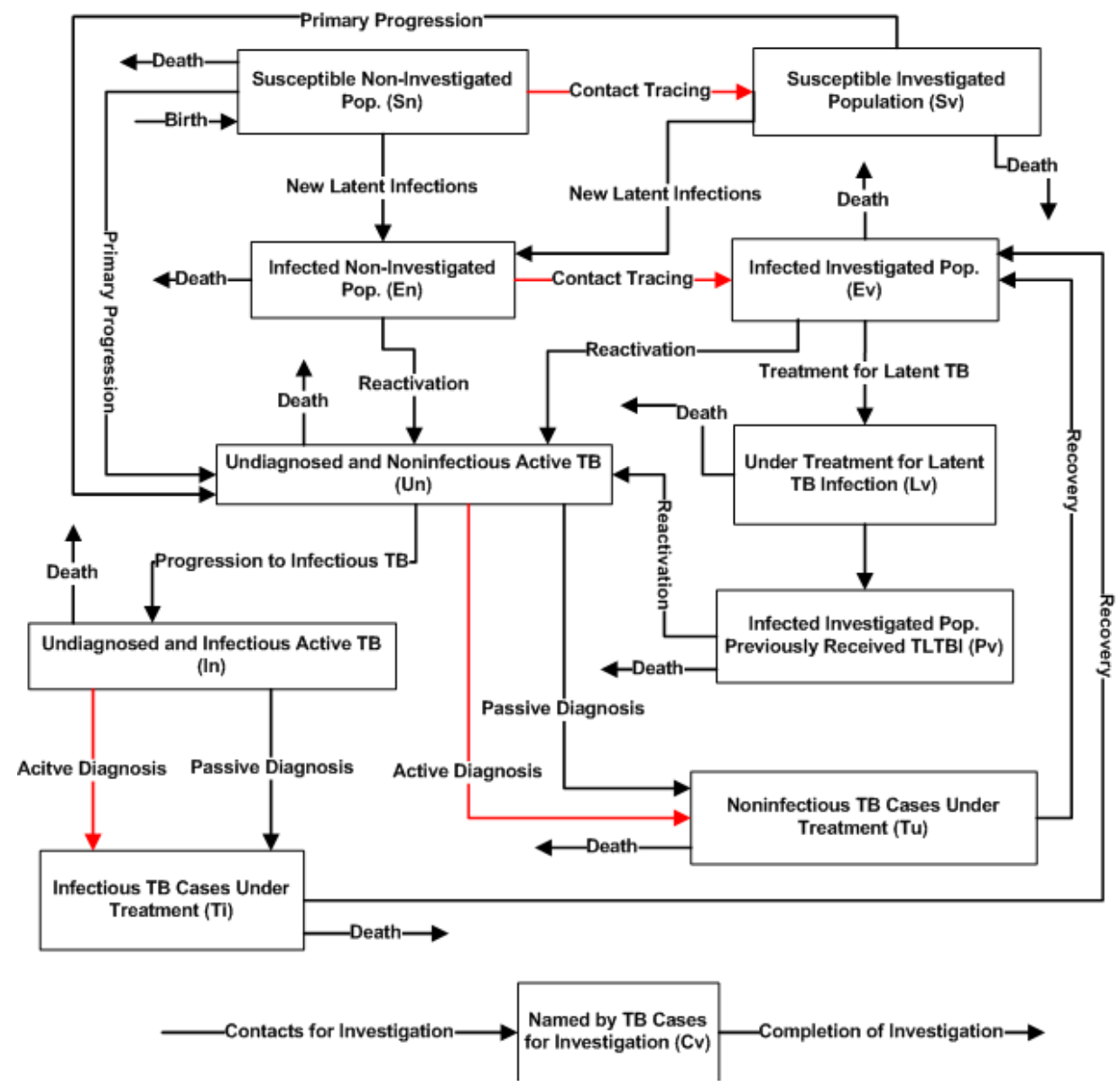

Figure 1: Structure of TB Model With Respect to Contact Tracing. 


\section{Tian, Alawami, Al-Azem, Osgood, Hoeppner, and Dutchyn}

According to Figure 1, Susceptible people are divided into the "Susceptible Non-Investigated Pop.(Sn)" and "Susceptible Investigated Pop.(Sv)". Similar categorizing patterns have also been applied on infected people with no current disease. Here, the "Investigated" designation denotes those for whom public health authorities have a record of their TB infection status, while "Non-Investigated" represent people who might be uninfected, infected or active TB cases, but where those authorities do not know their TB infection status. The investigation process is accomplished via a contact tracing program and is supported use of the Mantoux skin test to test whether an individual is infected by TB. Individuals who are susceptible whether investigated or not - can acquire TB infection by contact with an infectious cases; once infected, they will move into the "Infected Non-Investigated Pop.(En)" stock via persistently latent infection or "Undiagnosed and Noninfectious Active TB (Un)" via primary progression. After acquiring TB infection, those previously investigated susceptible people are no longer considered investigated, since their TB infection status has been changed and we cannot recognize these newly updated TB status unless they are investigated another time. Suppose a person is traced and found to be Mantoux negative; he will then remain in the "Susceptible Investigated Pop.(Sv)" stock. If this person is subsequently infected and becomes a latent TB case, he will go to the "Infected Non-Investigated Pop.(En)" stock. The reason why his investigation status goes to "non-investigated" is that, his current Mantoux test result which following infection should be Mantoux positive but not Mantoux negative - is as yet unknown to those performing contact tracing. And those authorities were not aware that he is infected. His investigation status will not be updated to "investigated" unless he is traced and tested for a second time.

People in "Infected Non-Investigated Pop.(En)" can either move into "Infected Investigated Pop.(Ev)" via contact tracing, or progress to the active TB disease state. Those infected and investigated people may undergo a prophylactic treatment (treatment for latent TB infection) for 6 months to lower their risk of progression to active TB disease. Not every infected and investigated individual can get TLTBI, because the eligibility of TLTBI is restricted by certain criteria, including those considering age, health status, history of Active TB and so on. The "Under Treatment for Latent TB Infection (Lv)" and "Infected Investigated Pop. Previously Received TLTBI (Pv)" stocks characterize those traced infected people who are currently under the treatment for latent TB infection (TLTBI) and those who have finished their prophylactic treatment and are currently under certain protection from developing disease. And it is assumed that TLTBI confers a 68\% efficiency in lowering the risk of progression to active TB (Osgood et al. 2011).

People can progress to active TB via either primary progression or reactivation. In our model, we distinguish people with active TB disease into 4 stocks according to their diagnosis status and TB infectiousness, namely "Undiagnosed and Noninfectious Active TB (Un)", "Undiagnosed and Infectious Active TB (In)", "Noninfectious TB Cases Under Treatment (Tu)" and "Infectious TB Cases Under Treatment (Ti)". Those undiagnosed noninfectious TB cases might develop infectiousness within a period of time if they are not diagnosed. It is worth emphasizing that both passive and active diagnosis methods are implemented in our model to highlight the role of contact tracing in identifying active TB cases, particularly those with the infectious form of active TB. After recovery from either the infectious TB state or the noninfectious TB state, they will return to the "Infected Investigated population" stock because authorities will know that their Mantoux test results remain positive.

The stock "Named by TB Cases for Investigation $(\mathrm{Cv})$ " represents the number of contacts that are queued for tracing and testing. Contacts who are named within this stock will be found to be infected investigated people (Mantoux Positive with no current disease), Susceptible investigated people (Mantoux Negative) or active TB cases through active diagnosis.

Every year, a number of infectious or noninfectious TB cases will be diagnosed. For those diagnosed as infectious TB cases, all of them will be traced. However, for those diagnosed as noninfectious TB cases, only a fraction of them - typically the primary TB cases - will be traced. Using average contacts per active TB cases per year, we can derive the average number of contacts traced per year. 


\subsection{Parameterization}

Values of parameters used in the model are either derived from the Saskatchewan TB Control Program database and provided supplemental information, and a variety of literature (Hasmiller 2007; Mahamoud et al. 2009; Osgood et al. 2011). Key parameters used in the model are depicted in Table 1.

Table 1: Description of the Symbols and Parameter Settings in TB Model with Contact Tracing

\begin{tabular}{l|l|l}
\hline Parameter & Description & Value \\
\hline$\beta$ & $\begin{array}{l}\text { Likelihood of infection per contact between a suscepti- } \\
\text { ble and an infectious active TB case }\end{array}$ & 0.46 \\
\hline$c$ & $\begin{array}{l}\text { Average number of contacts per TB case per unit time } \\
\text { (year) }\end{array}$ & $\begin{array}{l}40.96 \text { persons per } \\
\text { case }\end{array}$ \\
\hline$p_{r}$ & $\begin{array}{l}\text { The efficacy of TLTBI when measuring the risk of } \\
\text { developing active TB }\end{array}$ & 0.68 \\
\hline$m_{t}$ & $\begin{array}{l}\text { The mean time spent under treatment for active TB } \\
\text { cases }\end{array}$ & 0.75 year \\
\hline$m_{n}$ & $\begin{array}{l}\text { The mean time spent under TLTBI treatment for la- } \\
\text { tently infected people }\end{array}$ & 0.5 year \\
\hline$m_{i n}$ & $\begin{array}{l}\text { Mean time for a non-infectious TB case developing in- } \\
\text { fectiousness }\end{array}$ & 1.29 years \\
\hline$m_{c t}$ & $\begin{array}{l}\text { The mean time to trace and investigate a given contact } \\
\text { of that case (averaging over all contacts) }\end{array}$ & 0.25 year \\
\hline$\mu_{t b}$ & Mortality rate from TB & 0.037 per year \\
\hline$\mu_{n}$ & Mortality rate from other disease & 0.0274 per year \\
\hline$D$ & Rate of reactivation & 0.003125 per year \\
\hline$R$ & $\begin{array}{l}\text { Proportion of newly infected individuals progressing to } \\
\text { primary TB }\end{array}$ & 0.05 \\
\hline$K$ & Fraction of infected TB cases eligible for TLTBI & 0.3 \\
\hline$C_{t n}$ & $\begin{array}{l}\text { Average contacts per traced noninfectious TB cases per } \\
\text { year }\end{array}$ & 13.44 persons \\
\hline$C_{t i}$ & Average contacts per traced infectious TB case per year & 54.625 persons \\
\hline$Z$ & $\begin{array}{l}\text { Chance that an noninfectious TB case (particularly } \\
\text { primary TB case) been traced }\end{array}$ & 0.18 \\
\hline &
\end{tabular}

\subsection{Calibration}

Although the model includes many parameters values estimated from the relevant datasets and literature, there are still some parameters which can't be retrieved or easily estimated. Most notably, there are a large number of people with unknown/non-investigated TB status in our model. Several studies provided us with some rough estimates of the real world situation, such as data from mass screening for TB in Saskatchewan during the 1970 s and earlier. But it is only a rough estimation, and it is likely to exhibit pronounced variation.

In order to improve the validity and reliability of our model, it is highly valuable to calibrate our model to best match historically observed values between 2001 and 2007. These historical data across the time series include incidence rate, number of traced people with Mantoux positive results, and the population size of community 1. Calibration was performed using a Powell Optimization algorithm (used within Vensim software) (Vickers and Osgood 2010) to adjust several parameter values so as to find the best observed match to the historic data. The parameters to be calibrated in our model are the birth rate, initial fraction of TB infection, initial undiagnosed noninfectious TB cases and initial undiagnosed infectious TB cases. Firstly, birth rate was calibrated via setting its initial value as well as its upper and lower boun- 
daries. Then, with use of the calibrated birth rate, the remainder of the three parameters are calibrated by matching incidence rate and traced people with Mantoux positivity status. Each calibration is simulated for around 100,000 times to optimize a weighted sum of square about the discrepancies between the historical data and simulated results. The best calibrated parameter values we can get are shown in Table 2 .

Table 2: Calibrated and Estimated Symbols and Parameters

\begin{tabular}{l|l|l}
\hline Parameter & Description & Value \\
\hline$A$ & Annual birth rate of community 1 & 0.0469 per year \\
\hline$\pi$ & $\begin{array}{l}\text { Fraction of TB Infection. Infected people with no current disease } \\
\text { people over the total susceptible and infected people in community 1 } \\
\text { at 2001 }\end{array}$ & 0.286 \\
\hline$m_{d n}$ & $\begin{array}{l}\text { Mean Time until diagnosis for non-infectious active } \\
\text { TB }\end{array}$ & 0.6413 year \\
\hline$m_{d i}$ & Mean Time until diagnosis for infectious active TB & 0.5 year \\
\hline Initial In & Initial value of undiagnosed and Infectious Active TB stock & 1 person \\
\hline Initial Un & Initial value of undiagnosed and noninfectious Active TB stock & 1 person \\
\hline
\end{tabular}

\section{SCENARIO DEFINITIONS}

The main focus of our model is to investigate the effect of contact tracing on capturing the source of infection as early as possible and in controlling the spread of the disease. In order to assist the process of policy formulation and decision making in disease control using the model, we have designed a variety of scenarios to answer two "what-if" questions, and each scenario is simulated from 2001 to 2030 to investigate the potential outcomes under different parameter settings.

- How would the benefits of contact tracing vary if contact tracing were to be carried out on various fraction of the contacts of infectious active TB cases (e.g. $10 \%, 20 \%, 50 \%$ or all contacts)? To assess the cost-effectiveness of tracing all contacts, compared to tracing only a subset such as those thought to be at high risk (e.g. by reasons of prolonged or intimate exposure), we frame the question in the context of a community whose identity is restricted for reasons of confidentiality (community 1) by asking what level of incidence of active TB would result from tracing an successively larger fractions of contacts of an infectious active TB. We firstly abstract away from the issue of differing levels of risk for different subsets of the contact population, and instead consider the impact of investigating different fractions of the contact population, assuming an equal distribution of risk across that population. What might the incidence rate be if contact tracing is not performed? How much improvement in disease control can be attributed to a contact tracing investigation? In addition, considering the risk in terms of the incidence rate of active TB, we also explore the impact of partial contact tracing on prevalence of TB infection across the overall population.

- If we improve the speed of contact tracing, what would the impact be on TB incidence rate and prevalence over the time? Tracing contacts more promptly provides an opportunity to reduce exposure time and new TB infection cases. If we go a step further, lowering the number of newly infected people can eventually reduce the prevalence of TB infection as well as the incidence rate of active TB. Considering the highest level of population health, it is natural to seek to operate contact tracing investigation as fast as we can, nevertheless we are bounded by a limitation of human resources and budgets. A step towards understanding the cost-benefit trade-off of contact tracing speed is to evaluate the benefits of speedier contact tracing. If public health authorities can deploy sufficient resources to reduce the mean time of contact tracing by a factor of 10 or even of 20 , what attributable level of benefit would be secured? How many new TB infection cases can be eliminated? Our TB model seeks to explore these potential gains in the context of TB transmission. 


\subsection{Sensitivity Analysis on Coefficient of Tracing Contacts}

This experiment is designed to answer question 1 about the impact of tracing partial contacts of active TB cases. As a first step, we set our calibrated baseline model to represent ongoing contact tracing. For the calibrated baseline model, the coefficient of investigated contacts is 1 (or 100\%). Then based on this calibrated baseline model, we adjusted the coefficient of contacts coming into investigation to $0 \%, 10 \%, 50 \%$ and $150 \%$ of that baseline value. Coefficient with value of $0 \%$ indicates that there are no contact tracing ongoing within community 1 , while $150 \%$ means we spend extra effort in the interview session to identify additional contacts, with an additional 50\% margin of those reported contacts coming into investigation. This analysis is based on the assumption that people might not report their full social networks and contacts due to difficulties in recollection or personal reasons, and the response rate can hardly be $100 \%$, since contacts might have problems coming into investigation due to personal mobility or other reasons.

\subsection{Sensitivity Analysis on Mean Time of Tracing Contacts}

Within this scenario, we sought to investigate the effect of accelerating or decelerating the contact tracing process on TB control as a whole. For this experiment, we conducted simulations with different mean time for contact tracing including both speeding up and slowing down by a factor of 10 or even of 20. For this experiment, the coefficient of investigated contacts remained 1 for all experiments.

\section{EXPERIMENTAL RESULTS}

\subsection{Results of Coefficient of Tracing Contacts}

Prevalence of Active TB

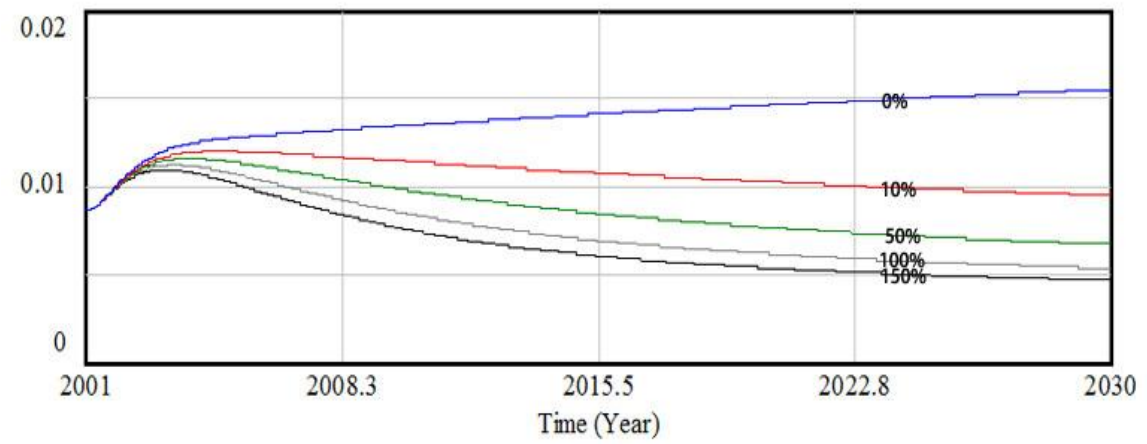

Prevalence of Active TB : Contact Tracing Coefficient $=\%$ (No contact Tracing)

Prevalence of Active TB : Contact Tracing Coefficient $=10 \%$

Prevalence of Active TB : Contact Tracing Coefficient $=50 \%$

Prevalence of A ctive TB : Contact Tracing Coefficient $=100 \%$ (Baseline)

Prevalence of A ctive TB : Contact Tracing Coefficient $=150 \%$

Figure 2: Prevalence of Active TB Corresponded with Different Coefficients of Investigated Contacts

Figure 2 presents the results for scenarios examining changes to the assumptions regarding the breadth of contact tracing. Absent contact tracing, the model suggests that the prevalence of active TB will be increasing over time. However, for scenarios with contact tracing, the prevalence of active TB declines over time, following a peak at roughly 2003. Tracing contacts at $10 \%$ of the baseline level shifts the increasing trend of prevalence into a decreasing one after a short period of increasing between 2001 and 2003. When tracing $50 \%$ of the baseline level, the prevalence of active TB decreases dramatically. When tracing at $100 \%$ of the baseline level, although the prevalence of active TB is lower than that resulting from tracing at $50 \%$ of that level, the incremental benefits are very limited. In short, there is a much larger reduction in prevalence secured by tracing the first $50 \%$ of the baseline level of contact tracing than is obtained by 
tracing at $50 \%$ of the baseline level. Similar patterns can also be identified when coefficients of contact tracing is $100 \%$ and $150 \%$. Tracing extra $50 \%$ of the baseline level doesn't gain much benefit.

Incidence Rate

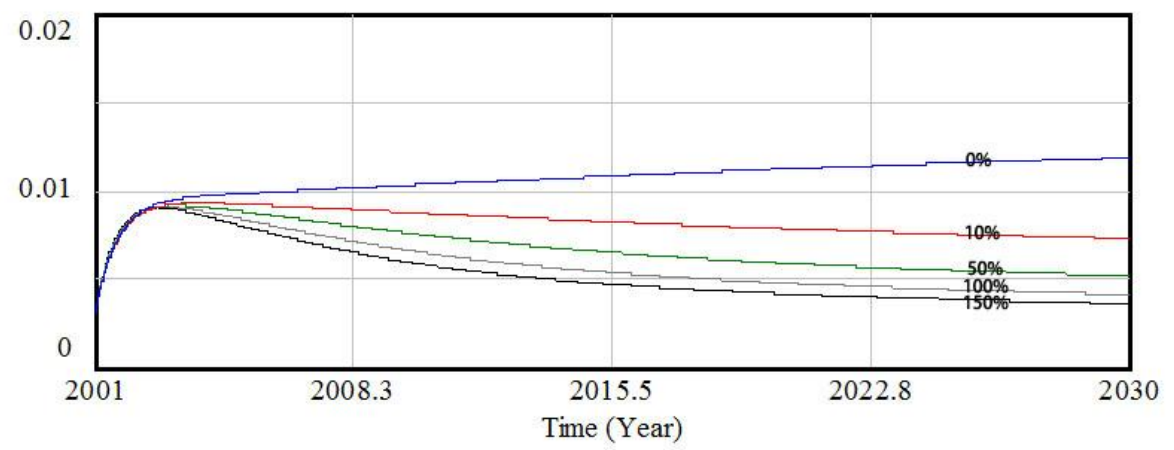

Incidence Rate : Contact Tracing Coefficient $=0 \%$ (No contact Tracing)

Incidence Rate : Contact Tracing Coefficient $=10 \%$

Incidence Rate : Contact Tracing Coefficient $=50 \%$

Incidence Rate : Contact Tracing Coefficient $=100 \%$ (Baseline)

Incidence Rate : Contact Tracing Coefficient $=150 \%$

Figure 3: Active TB incidence rate for different coefficients of investigated contacts

Figure 3 shows the impact of additional levels of contact tracing on the incidence rate for Active TB. All scenarios initiate with a transient consisting of a sharp rise in the incidence rate. This transient suggests an inconsistency between factors within the model, such as between of the fraction of infection assumed within the initial population on the one hand, and the rates of mixing (c) and transmissibility of the bacterium within this epidemiological context $(\beta)$. Absent contact tracing, the active TB incidence rate increases slowly following the initial transient. When tracing at $10 \%$ of the baseline level, the incidence rate lies below that resulting from no contact tracing with a declining trend. When tracing at $50 \%$ of the baseline level, the incidence rate declines slowly over time after reaching its peak around in 2003 . The incidence rate decreases faster after 2002 when tracing more contacts, but once again the extra benefits we obtain when tracing incrementally larger fractions of contacts (compared to the baseline) yield markedly decreasing returns. For example, the benefits gained when tracing at 50\% larger population than is traced at the baseline (compared to the benefits of no contact tracing) is far larger than the incremental benefit tracing of at $100 \%$ of the baseline level (compared with tracing at 50\% of that level). Similarly, tracing an extra $50 \%$ increase in the rate relative to the baseline scenario contributes even smaller benefit.

\subsection{Results of Mean Time of Tracing}

This experiment demonstrates the impact of reducing the mean time of contact tracing on the TB control, as judged by the prevalence of active TB. According the trend lines displayed in Figure 4, as a whole all the trajectories have the declining tendency over the time, except for the initial transient. We observe that changing the mean time of contact tracing slightly around the baseline doesn't provide pronounced benefits in TB control. The impact of decreasing the mean time of contact tracing even by integral factors (e.g. a factor of 2 or 3 ) is very small, and for small changes, the impact can almost be ignored. Even for larger changes (such as the factors of 10x shown in the diagram), the impacts are very limited. While the aggregate character of the model suggests the need for great caution, such results suggest that there may be limited gains to be secured by investments in reducing the time required for contact tracing.

Figure 5 illustrates the fraction of actively diagnosed cases among all the incident cases over time. As a whole, all the trajectories increase first and decrease afterward. We observe that the faster contact tracing investigation goes, the sooner the fraction of the actively diagnosed cases reach its peak. 


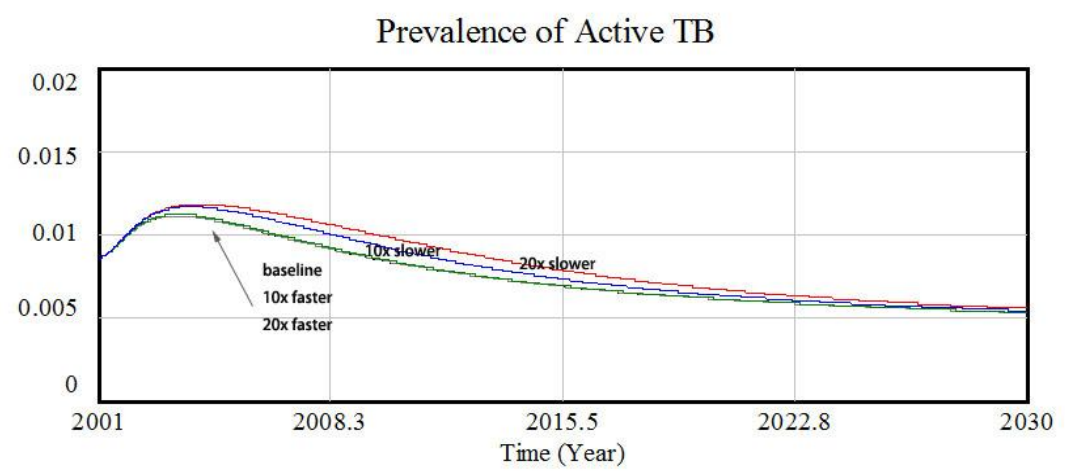

Prevalence of Active TB : Mean Time of Tracing 10x slower Prevalence of A ctive TB : Mean Time of Tracing 20xs slower

Prevalence of Active TB : Mean Time of Tracing Original (baseline)

Prevalence of Active TB : Mean Time of Tracing 10x faster

Prevalence of Active TB : Mean Time of Tracing 20x faster

Figure 4: Prevalence of active TB produced by different mean time of tracing contacts

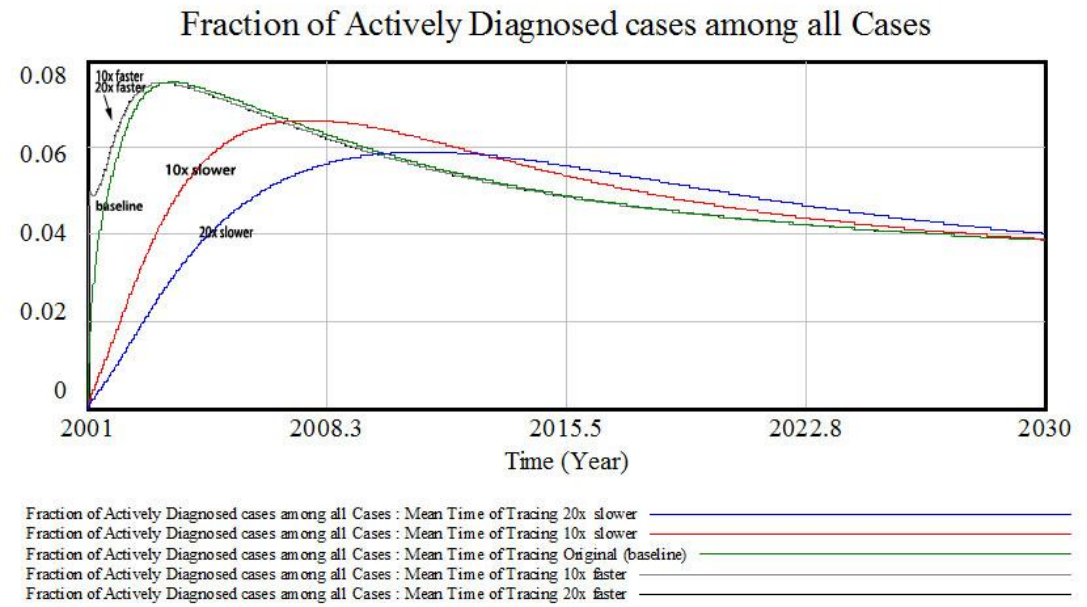

Figure 5: Fraction of Actively Diagnosed Cases Among All Incident Cases

\section{DISCUSSION AND CONCLUSION}

The first question explored is this paper concerned the impact of tracing partial contacts on disease control and prevention. In this area, our models have explored a few alternatives focused on tracing subsets of the contacts. Model results suggest that the impact of contact tracing is pronounced in TB control as we have observed dramatic decrease in incidence rate and the prevalence of active TB. For the scenarios exploring partial tracing, we found that incidence rate decreases when tracing successively larger fractions of contacts; however, the contribution of tracing more contacts declines markedly. This phenomenon of diminishing returns would appear of relevance when developing control policies. The results suggest that strong gains can be obtained when tracing merely partial contacts. Given limited resources, it also tentatively suggests that tracing partial contacts may be more effective and beneficial especially when we set priority for contact tracing to those with high risk (such as those experiencing longer exposure times, or individuals who are known to have experienced past contacts with several other cases).

Our second investigation sought to understand the mean time of contact tracing. Within this area, the model was used to explored a few scenarios. Given model assumptions, TB control effectiveness is relatively insensitive to mean time of contact tracing especially within the large range of 20 times faster to 20 


\section{Tian, Alawami, Al-Azem, Osgood, Hoeppner, and Dutchyn}

times slower than the baseline. When reducing the mean time of contact tracing and boosting the effort to track TB cases sooner compared to the baseline, the achievement yields only modest gains and the patterns are similar to that obtained at baseline. This suggests that there may be unexpected degrees of flexibility in the speed of tracking contacts, and raises the interesting possibility that the current guidelines and schedule for contact tracing are sufficient fast to secure most of the benefits to be gained through contact tracing.

By contrast, through experiments with different fractions of contacts coming into investigation, it is found that assumptions regarding the fraction of contacts coming into investigation yield pronounced impacts for the prevalence of TB infection. Bringing larger fractions of contacts of individuals in for contact tracing can yield a reduced prevalence of TB infection. Tentative though they are, the results suggest that the gains exhibit diminishing returns. Depending on the relative cost of undertaking contact tracing and the cost of treating TB, putting into place some basic measure of contact tracing would appear likely to yield the greatest incremental benefit (when compared with enhancing the level of tracing experienced in a program already drawing many contacts).

While deducing the optimal level of contact tracing will require the consideration of the cost tradeoffs involved. Such diminishing returns suggest that the cost-effectiveness view, there is likely to be decreasing cost-effectiveness for bringing in larger sets of individuals. While the current model did not seek to capture important known risk factors for TB, such observations would suggest that focusing limited contract tracing effort on a smaller segment of all contacts who have a very high risk of being infected or of developing active TB given infection could yield disproportionate benefit. Such high-risk individuals would include contacts who are known to have had contacts with many other cases, and those in the top risk categories currently used by TB control. With priority setting in tracking the contacts, the striking gains from partial contact tracing seen here will likely be significantly more efficient yet.

We were surprised to find such limited incremental effectiveness due to reductions in the time required for performing contact tracing. While this phenomenon merits close additional study before any definitive policy-relevant implications are drawn, it raises the provocative possibility that the current speed of contact tracing may be securing the large majority of the benefits that would be gained even by far faster contact tracing. It is undeniable that speeding up the contact tracing process would offer some benefits, but in the context of limited health resources (funds and working staff), model results raise the distinct possibility that the benefits from such speed-ups may be less than the associated opportunity cost.

At a more fundamental level, model experimentation with the scenario results suggests that, when viewing the situation from a cost-effectiveness standpoint, it is important to recognize that contact tracing is, in a sense, "self-limiting". This effect is particularly notable with respect to the speed of contract tracing. Initially, rapid contact tracing will quickly reduce the incidence - and, more slowly, the prevalence of active TB. The more rapid is the contact tracing involved, the sooner will these rates fall. However, whether these gains are achieved sooner or later, the results tend to converge. This convergence reflects the fact that reduced Active TB prevalence in the population limits the efficiency of contact tracing by imposing larger numbers of contacts to be traced before a case of infectious TB can be located. It is entirely possible that find an active TB cases need to test hundreds of contacts which need lots of resources and money. As a result, regardless of how quickly the prevalence of active TB initially decreases, these initial decreases will slow significantly due to the inefficiency of contact tracing, and will converge to similar levels of prevalence (an effect seen clearly in Figure 4 and Figure 5).

At this point, the greatest gains in efficiency may be secured by effective risk prioritization, which could allow the given investment in resources to secure significant additional benefit (Al-Azem, Hoeppner, and Osgood 2010). By contrast, while its dependence on passive methods of diagnosis (in which an infective individual presents for care, typically as a result of symptoms) can allow significant reservoirs of infective cases to circulate in the population, the efficiency of passive diagnosis is less adversely affected by decreasing prevalence of TB than is contact tracing with an important exception being the reduced likelihood that a given physician will recognize TB within a population in which TB circulates at low levels. In this sense, contact tracing can easily become a "victim of its own success", with its initial suc- 


\section{Tian, Alawami, Al-Azem, Osgood, Hoeppner, and Dutchyn}

cessful reduction of the prevalence of TB tending to reduce over time the fraction of all active TB cases that are brought in via active diagnosis, and over time shift larger burden of case-finding to passive methods. It is important to emphasize that a situation in which low fraction of case finding occurs due to active methods of diagnosis, should not automatically be taken as an indictment of the limited efficiency of contact tracing per se. It may well be the case that the presence of contact tracing processed is what has allowed for the lowering of prevalence to the current point, and the maintenance of the system at low levels of active TB. In so doing, it is likely that it will make itself appear "inefficient" to the amount of effort entailed in finding new infectious cases - but this apparent "inefficiency" belies the key role of contact tracing in achieving the current situation.

While the preliminary and aggregate character of the model suggest the need for great caution in interpreting model results in a quantitative fashion, the simulation outcomes suggest some very tentative public health related implications in disease control and policy formulation. This study has focused on contact tracing investigation within the context of an aggregate simulation model. While simulation of contact tracing can very naturally be carried out at an individual level, System Dynamics modeling can assist in the process of policy optimization by running different scenarios to predict potential control outcomes. Modeling within this area raises the potential for maintaining more judiciously chosen programs and enhancing the reliability and validity of deploying new prevention strategies.

However, the model described in this paper exhibits significant drawbacks. Firstly, our implementation of contact tracing ignores the underlying contact patterns within the population. Due to the nature of the aggregate model, it cannot readily be used to investigate existing or prospective alternative contact tracing protocols, because it deals with people in an aggregate way rather than as individuals. In addition, the model does not capture the population heterogeneity in risk across the population. Among other factors, the assumption of a homogeneous population limits our capacity to investigate targeted contact tracing strategies, which could be highly important for enhancing the efficiency of contact tracing. We are currently working to address this shortcoming using simulations on individual-based model with network structure. An individual-based model would provide a natural vehicle for examining the impact of expediting contact tracing according to the levels of risk factors. A further limitation of the current model is the failure to represent BCG. Although we recognize the potential importance of protection conferred by BCG, we have not represented its impact on preventing TB infection in our model for several reasons. The first of these reasons is limited data: Many individuals in the TB database employed lack BCG information recorded in their files. In addition, the BCG data that is available is imprecise: Most traced individuals have limited recollection as to whether or not they underwent inoculation with BCG. Finally, recognizing the considerable variability in the epidemiological data on BCG efficacy (Ward 2004), the degree of efficacy of the BCG vaccine is unclear. In this regard, it is notable that some researchers argue that the vaccine confers little or no benefit within some populations, such as those in higher latitudes (Bretscher 1992).

\section{REFERENCES}

Al-Azem, A. 2007. "Social Network Analysis in Tuberculosis Control Among the Aboriginal Population of Manitoba." PhD dissertation, University of Manitoba, Canada.

Al-Azem, A., V. Hoeppner, and N. Osgood. 2010. "Social Network Analysis (SNA) advantages in tuberculosis (TB) control in high TB incidence community in Saskatchewan." In Proceedings INSNA Sunbelt $X X X$. Trento, Italy. July, 1.

Anderson, R. M., and R. M. May. 1991. Infectious diseases of humans: dynamics and control. Oxford and New York: Oxford University Press. ISBN 0198545991.

Aparicio, J.P., and J.C. Hernandez. 2006. "Preventive treatment of tuberculosis through contact tracing." Contemporary Mathematics 410.

Bretscher PA. 1992. "A strategy to improve the efficacy of vaccination against tuberculosis and leprosy." Immunology Today 13 No. 9:342-345. 
Eames, K. T. D., and M. J. Keeling. 2003.“Contact tracing and disease control.” In Proc. R. Soc. Lond. B 270, 2565-2571.

Hassmiller, K. 2007. "The impact of smoking on population-level tuberculosis outcomes." Ph.D. thesis, University of Michigan. USA.

Huerta, R., and L. S. Tsimring. 2002. "Contact tracing and epidemics control in social networks". Phys. Rev. E 66 No. 5, article number 056115.

Hyman, J. M., J. Li, and E. A. Stanley. 2003. "Modeling the impact of random screening and contact tracing in reducing the spread of HIV". Math. Biosci. 181:17-54.

Mahamoud, A., Y. Liu, et al. 2009. "Tuberculosis Modeling Project: Reconstruction of Kristen M. Hassmiller's Model." Course report, Department of Computer Science, University of Saskatchewan.

Osgood, N. D., A. Mahamoud, K. H. Lich, Y. Tian, A. Al-Azem, and V. H. Hoeppner. 2011. "Estimating the relative impact of early-life infection exposure on later-life tuberculosis outcomes in a Canadian sample". Research in Human Development 8 No. 1:26 - 47.

Vickers, D. M., and N. D. Osgood. 2010. "Current crisis or artifact of surveillance: Insights into rebound Chlamydia rates form dynamic modeling." BMC Infect. Dis. 10:70.

Ward, H. A. 2004. "Risk Factors in the Progression from Tuberculosis Infection to Disease". Master dissertation, University of Saskatchewan, Canada.

White, P. J., H. Ward, J. A. Cassell, C. H. Mercer, and G. P. Garnett. 2005. "Vicious and virtuous cycles in the dynamics of infectious diseases and the provision of health care: gonorrhea in Britain as an example." J Infect Dis. 192:824-36.

Ziv, E., and C. L. Daley, and S.M Blower. 2001. "Early therapy for latent tuberculosis infection." Amer. J. of Epidemiology 153 No. 4:381-385.

\section{AUTHOR BIOGRAPHIES}

YUAN TIAN (yut473@mail.usask.ca) is an M. Sc candidate in the Dept. of Computer Science at Univ. of Saskatchewan. She works on System Dynamics and Agent-based modeling of infectious disease.

FATIMA ALAWAMI (faa359@mail.usask.ca) is an M. Sc candidate in the Dept. of Computer Science at Univ. of Saskatchewan. She works on Aspect refactoring tools.

ASSAAD AL-AZEM (aaa823@mail.usask.ca) is a clinical epidemiologist working in the Dept. of Medicine at Univ. of Saskatchewan. His work involves social network analysis in TB Control.

NATHANIEL OSGOOD (osgood@cs.usask.ca) is an Associate Professor in Dept. of Computer Science at Univ. of Saskatchewan. His research is focused on providing tools to inform understanding of population health trends and health policy tradeoffs.

VERNON HOEPPNER (vernon.hoeppner@usask.ca) served as the director of Saskatchewan Tuberculosis Control for over 30 years, and currently the Department Head of the Dept. of Medicine at Univ. of Saskatchewan.

CHRISTOPHER DUTCHYN (dutchyn@cs.usask.ca) is an Assistant Professor in the Dept. of Computer Science. Most of his work is on language semantics and implementation, focusing on aspect-oriented languages in particular. 